\title{
Effect of Stall Strip Position, Size and Geometry on the Lift Coefficient of NACA 001 Aerofoil
}

\author{
Wee Tee ${ }^{l}$, Ahmed Nurye Oumer ${ }^{l}$, Ahmmad Shukrie $^{l}$, Azizuddin AbdulAziz ${ }^{1}$, and Aklilu \\ Baheta $^{2}$ \\ ${ }^{1}$ Faculty of Mechanical Engineering, Universiti Malaysia Pahang, 26600 Pekan, Malaysia \\ ${ }^{2}$ Faculty of Mechanical Engineering, UTP, 32610 Seri Iskandar, Malaysia
}

\begin{abstract}
This study aims to determine the optimum position and geometry of stall strips (SS) to control sudden fall of lift in wind turbine blades. The type of airfoil used in this study is NACA 0015 with $150 \mathrm{~mm}$ of chord length. Total of five positions, two geometries and three sizes of SS configurations are simulated by using Ansys Fluent software. For position configuration, SS of size $2 \mathrm{~mm}$ is placed on the apex (POS-1), and on the upper and lower surfaces at distance of $0.65 \mathrm{~mm}$ (POS-4 and POS-2 respectively), and $2.45 \mathrm{~mm}$ (POS-5 and POS-3), respectively, from the leading edge. The shapes tested are dome and equilateral triangle. The results show that the addition of SS as a method of controlling sudden loss of lift decreases the maximum lift coefficient. Attachment of SS at the lower surface of the airfoil did not bring any significant effect to the lift and stall characteristics; while for the upper surface it reduces the sudden fall of lift but at the cost of big reduction in maximum lift coefficient. The optimum position and geometry of SS are POS-1 and triangle shape. Increasing in size of SS shows positive effect in control stalling effect.
\end{abstract}

\section{Introduction}

Aerodynamic ability is the main criteria in designing shape of wind turbine blades. The lift coefficient of an airfoil increases with the increase in angle of attack (AOA). Beyond critical AOA, lift coefficient drops significantly due to stalling effect. The design of shape of airfoil is essential to provide maximum lift and minimum drag of turbine blade without inducing major stalling effect, directly affecting performance of a wind turbine. Lift modifying devices have been used on wind turbine blades to improve the lift/drag ratio or otherwise modify the stalling characteristics of the blade. The addition of these devices on airfoil bring effects on lift and drag, and trade-offs between lift and drag are usually expected [1]. Stall strip (SS) is a lift modifying device usually triangle in shape, installed to the leading edge of airfoil in wind turbine and aircraft. Installation of stall strips serve the purpose of modifying the streamline flow around the airfoil so that the stalling effect is altered [2].

Various research works have been done on the application of stall strips for improved performance of turbine blades and airfoils [3-5]. Experiment on wind tunnel test of NACA 63-415 attached with stall strips was done by Christian et al. [6]. Stall strips are positioned at different location to analyze the effects on lift coefficient. His results show that 
aerodynamic coefficients are most significantly affected when stall strips are attached at stagnation position of $-2^{\circ}$. Maximum lift decreased from 1.33 to 1.00 while minimum drag increased to 0.010 from 0.008 . The effect of SS to the aerodynamic characteristic of airfoil can be ignored when mounted beyond stagnation point $10^{\circ}$ AOA [6]. Zahle et al. [7] investigated the same type of airfoil by using 2D computational analysis. The simulation when compared to experimental dome by Christian, shows similar results. However, the author did not make any conclusion due to insufficient agreement with the experimental data. In separate study, Lewis et al. [8] conducted experiment to determine the optimum position and size of SS on NASA LS (1)-0417 MOD airfoil. From the experiment, SS were found to be most effective at position close to the base line. At this location, SS of height 3 $\mathrm{mm}$ provided drag control without inducing stalling effect. The author concluded that using SS to control stalling effect has a limitation which is not possible to increase the lift coefficient.

To the best knowledge of the authors this paper, the best location and geometry of the SS is not fully understood. Thus, the main objective of this project is to determine the optimum position and geometry of SS on the leading edge of airfoil to control sudden fall of lift in wind turbine blades. The additional objective is to study the effect of the size of SS towards the stalling characteristics of airfoil in wind turbine. The project is approached by 2D computational analysis method using ANSYS Fluent.

\section{Aerofoil and Stall Strip Geometries}

The type of airfoil used in the simulation was NACA 0015 with chord length of $150 \mathrm{~mm}$. The geometries of stall strips tested were equilateral triangle and dome shapes. Each geometry had three different sizes namely $2 \mathrm{~mm}, 3 \mathrm{~mm}$ and $4 \mathrm{~mm}$. The simulation was done by placing the stall strips at five different positions near to the leading edge of the airfoil. The SS of size $2 \mathrm{~mm}$ was tested at all position while SS of size $3 \mathrm{~mm}$ and $4 \mathrm{~mm}$ were tested at position POS-1 only. The chord-wise positions of the stall strips attached near the leading edge of the airfoil and their distance from the leading edge are shown in Table 1 .

Table 1: Positon of stall strips on airfoil

\begin{tabular}{|c|c|c|}
\hline Denotation & Chord-wise position (\%) & Distance from leading edge (mm) \\
\hline POS-1 & 0.000 & 0 \\
\hline POS-2 & 0.435 & 0.65 \\
\hline POS-3 & 1.635 & 2.45 \\
\hline POS-5 & 1.635 & 2.45 \\
\hline POS-4 & 0.435 & 0.65 \\
\hline
\end{tabular}

\section{Numerical Details}

\subsection{Governing Equations}

In this study, the flow field is assumed to be incompressible, steady and isothermal. Therefore, the governing equations for the continuity and momentum were solved together with turbulent equations. Thus, the governing equations are expressed as [9-13]:

$$
\begin{gathered}
\vec{\nabla} \cdot \vec{V}=0 \\
\rho[(\vec{\nabla} \cdot \vec{V}) \vec{V}]=-\vec{\nabla} P+\rho \vec{g}+\mu \vec{\nabla}^{2} \vec{V}
\end{gathered}
$$


where $\vec{\nabla}$ is divergence operator, $V$ is velocity vector of the fluid $(\mathrm{m} / \mathrm{s}), \rho$ is density of the fluid $(\mathrm{kg} / \mathrm{m} 3), P$ is the hydrostatic pressure $(\mathrm{Pa}), g$ is gravitational acceleration $\left(\mathrm{m} / \mathrm{s}^{2}\right)$ and $\mu$ in the fluid viscosity $(\mathrm{kg} / \mathrm{m} . \mathrm{s})$.

\subsection{Meshing and Boundary Conditions}

The mesh was generated by using ANSYS ICEM CFD and C-type grid topology was used. Sizing method with biased behavior was used to create finer grids at locations close to the airfoil walls where superior accuracy is needed. The objective is to obtain accurate result near the airfoil and reduce computational time. The flow in the simulation was considered to be laminar with Reynolds number around 100,000. Air was forced to enter to the computational domain from the left side at constant velocity, while the top and bottom walls are assigned as symmetry planes. The right side of the domain and the edges of the airfoil were assigned as outlet and wall, respectively. The properties of air such as density and viscosity were obtained at room temperature.

Most of the time in numerical simulation, increase number of mesh elements lead to better accuracy of result. However, increasing of element quantity requires extensive of computational time. Thus, proper mesh size should be selected for any computational domain. For this, mesh independence study was carried out to determine the optimum number of elements that are sufficient to produce accurate results. In order to provide accurate results, the grids around the airfoil were refined. It was observed that the values of $\mathrm{C}_{\mathrm{L}}$ are same for mesh sizes of 40,000 and above. Thus, mesh size of 40,000 is sufficient to deliver reliable results for the rest simulation works. Further increment of elements cost a lot of computational times and the improvement in accuracy is small-scale.

\subsection{Model Validation}

Before further analysis of the effect of stall strip on the performance of an airfoil, the accuracy and reliability of the developed model should be checked. For this, model validation was carried out to determine whether the developed model is suitable for analysis of drag and lift of airfoil without SS. The simulation results of clean NACA 0015 airfoil were compared with the results from published experimental data obtained using wind tunnel test [15]. Fig. 2 shows variation of lift coefficient, $C_{L}$ at various angles of attack obtained from the experimental data of Robert et al. and current simulation model. As we can see from the figure, the lift coefficient values of both experiment and simulation model have similar pattern. The pick value of $\mathrm{C}_{\mathrm{L}}$ for the experiment occurred slightly at lower AOA than the simulation one. The average percentage error between the current simulation results and experiment results is $6.1 \%$. Since the average error of lift coefficient is within the acceptable range, the results obtained from the simulation are considered to be correct.

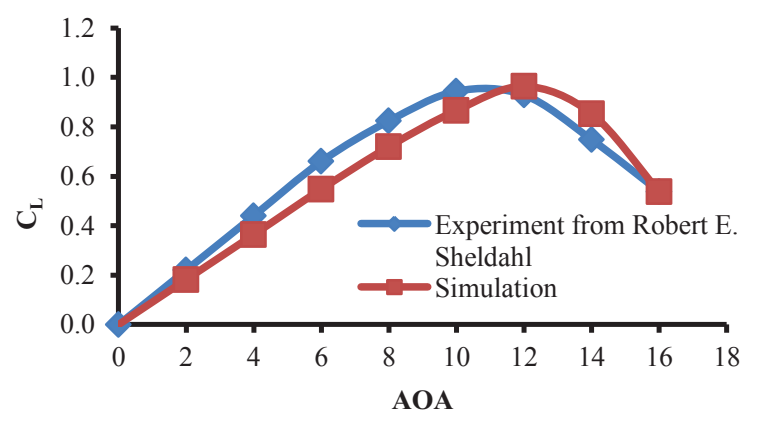

Fig. 1. Comparison of CL between simulation and experiment [15] at various AOA 


\section{Results And Discussions}

\subsection{Effect of SS Position on Lift}

Shown in Figures $2 \mathrm{a}$ and $2 \mathrm{~b}$ are variations of lift coefficient curves when dome and triangular shaped stall strips are added at five different positions. As can be clearly seen in Figure 2a, the stall strips placed at position POS-2 and POS-3 did not affect the lift and drag coefficients of NACA 0015 significantly. The highest lift coefficient was obtained for POS-3 around AOA $12^{\circ}$ and this value is equal to 0.982 ; while the clean airfoil had 0.964 . Placing of stall strips at POS-1 reduced the maximum lift coefficient to 0.84 and the AOA where this value obtained was at $11^{\circ}$. For position POS-4 and POS-5, the AOA for maximum $\mathrm{C}_{\mathrm{L}}$ were around $8^{\circ}$ to $10^{\circ}$, respectively, with maximum lift coefficient of 0.704 and 0.613 respectively. The lift coefficient trend rose back again after AOA $12^{\circ}$. At AOA $16^{\circ}$, the lift coefficient for all configurations converged to value between 0.482 and 0.562 . The effect of the SS is largely dependent on the formation of separation bubble. Introducing free-stream turbulence most likely alters the formation of such a bubble by increasing the mixing of the fluid around the SS.

Similar trends of $\mathrm{C}_{\mathrm{L}}$ were obtained for the case of triangular strips addition as shown in Figure $2 \mathrm{~b}$. Stall strips placed at POS-3 increased the maximum lift coefficient of NACA 0015 from 0.964 to 0.988 . However the curve gradient from $\mathrm{AOA} 12^{\circ}$ to $\mathrm{AOA} 14^{\circ}$ was increased. It indicates enhanced effect in sudden loss of lift. In POS-2, the maximum lift coefficient was dropped by $4.97 \%$ and did not show any improvement in controlling stalling problem. Placement of stall strips at POS-4 and POS-5 positions decreased the critical AOA from $12^{\circ}$ to $8^{\circ}$ and $6^{\circ}$ respectively. POS-4 and POS-5 showed positive effect in reducing stalling effect but maximum lift coefficient before stalling dropped drastically by $46.8 \%$ and $62.5 \%$, respectively. For POS- 1 , the critical AOA was reduced to around $12^{\circ}$ and magnitude in drop of lift coefficient beyond critical AOA was reduced.

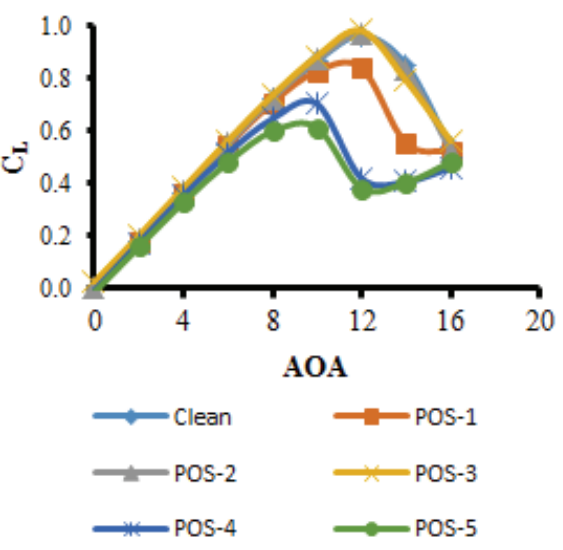

(a)

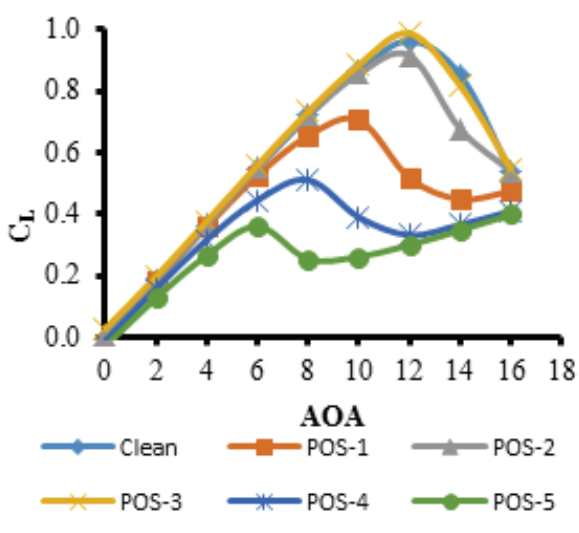

(b)

Fig. 2. Variation of $C_{L}$ curves when (a) dome shaped and (b) triangular shaped stall strips are added at five different positions.

\subsection{Effect of SS Shape on Lift}

The effect of stall strip shape towards the stalling characteristic of airfoil was brought to comparison. For this, lift coefficient results for clear airfoil and airfoils with doom shaped and triangular shaped stall strips attached at position POS-1 were compared as shown in 
Figure 3. For all geometries, there was no noticeable difference in lift coefficient curves at low angle of attacks (i.e., AOA $<6^{\circ}$ ). However, the difference is visible at high AOA with maximum lift coefficient occurring at angle of attack of $13^{\circ}, 12^{\circ}$, and $10^{\circ}$ for the clean, doom, and triangular stall strips respectively. Overall, the triangular shaped stall strip showed less sudden loss of lift effect, lower maximum lift coefficient and lower critical AOA than dome shaped SS. The curve gradients at the stalling region of triangular shaped SS were lesser while that of dome shaped had no significant difference with clean airfoil. On the other hand, the triangular shaped stall strip showed better results in controlling sudden loss of lift of the airfoil. The difference in lift coefficient started to converge after AOA $12^{\circ}$. At AOA $16^{\circ}$, all geometries had very close values of lift coefficient.

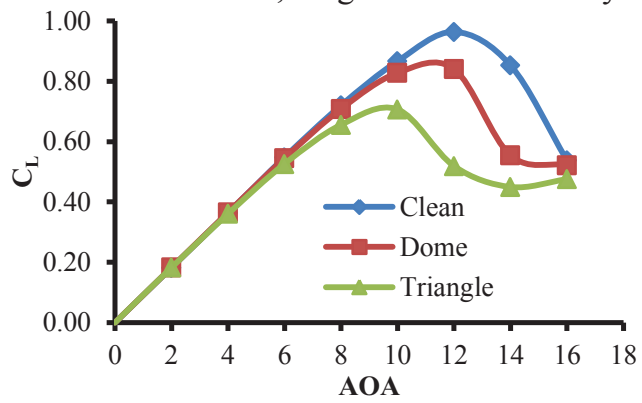

Fig. 3. Comparison of lift coefficient between clean airfoil and airfoil with dome and triangular shaped stall strips attached at positon POS-1

\subsection{Effect of SS Size on Lift}

Shown in Figures $4 \mathrm{a}$ and $4 \mathrm{~b}$ are variation of lift coefficient with stall strip size when dome and triangular shaped stall strips, respectively, are added at position POS-1. As can be clearly seen in Figure 4a, the lift coefficient curves for $2 \mathrm{~mm}, 3 \mathrm{~mm}$, and $4 \mathrm{~mm}$ stall strips remain almost the same with a slight increment from AOA $12^{\circ}$ onwards. The average increment of lift coefficient for $3 \mathrm{~mm}$ and $4 \mathrm{~mm}$ stall strips compared to the $2 \mathrm{~mm}$ one were only $0.67 \%$ and $3.25 \%$, respectively. It can be concluded that increasing in size of dome shaped stall strips did not improve the stalling behavior of the airfoil. Similarly, the effect of stall strip on the lift coefficient for triangular SS addition is not significant as shown in Figure $4 \mathrm{~b}$. However, the lift coefficient curves indicated that the increase in size of SS brings positive effect in controlling sudden stall effect. Increasing of size decreased the stalling effect as well as the maximum lift coefficient. Comparison between $4 \mathrm{~mm} \mathrm{SS}$ with $2 \mathrm{~mm}$ SS showed that the maximum lift decreased by $8.43 \%$ and the fall of lift from AOA $10^{\circ}$ to $\mathrm{AOA} 14^{\circ}$ decreased by $29.8 \%$.

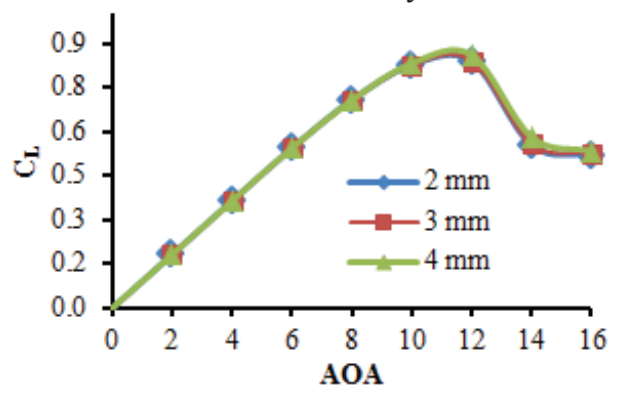

(a)

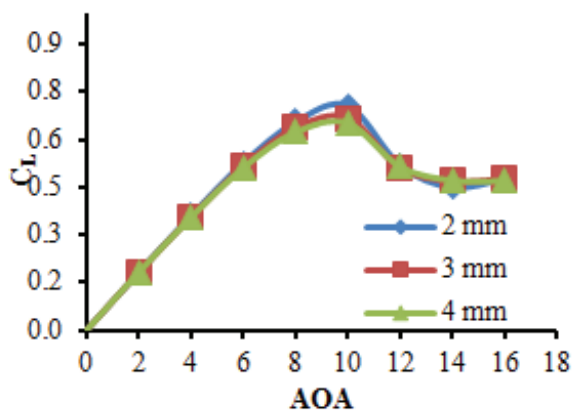

(b)

Fig. 4. Variation of lift coefficient with stall strip size when (a) dome and (b) triangular shaped stall strips are added at position POS-1 


\section{Conclusion}

In this research, total of five positions, two geometries and three sizes of stall strip configurations added on NACA 0015 airfoil were simulated. The results led to the a conclusion that addition of stall strips as a method to control sudden fall of lift in wind turbine blades sacrificed the maximum lift coefficient. The optimum (suitable) geometry of stall strip appeared to be triangular shaped SS. With triangular shaped stall strips, position POS-1 had the optimum sudden fall control to reduction of lift coefficient ratio. On the other hand, different geometries of the stall strips showed different effects to the lift coefficient. For triangular stall strips, increasing in size of stall strips did slightly reduce the sudden fall of lift. While for dome shaped stall strips, the increment of size of stall strips did not change the stalling effect. The findings from this project may help in future design and development of stall strip in airfoils.

\section{Acknowledgment}

The authors would like to thank Universiti Malaysia Pahang for providing necessary assistance for providing financial support under grant number RDU150375.

\section{References}

1. H. Stiesdal, P. B. Enevoldsen, Google Patents (2006).

2. D. Crane, Dictionary of Aeronautical Terms. $3^{\text {rd }}$ ed. (1997).

3. C. Amanullah, M. Arjomandi, and R. Kelso, Renew. Ener., 86: 26-37, (2016).

4. J.W. Lee, J.S. Lee, and J. H. Han,. Jour. of Wind Eng.and Ind. Aerodyn., 110: 62-69, (2012).

5. W. Geissler, G. Dietz, and H. Mai, Aerosp. Sci.e and Tech., 9(5): p. 390-399.

6. B. Christian, F. Peter, J. Jeppe, A. Ioannis, Riso Nat. Lab., Roskilde, Denmark, (2000).

7. F. Zahle, N.N. Sørensen, and J. Johansen, DTU Orbit - The Res. Info. Sys. (2002).

8. R. I. Lewis, I. Potts, and A. A. Arain,. 15: 40 (1995).

9. Y. A. Cengel, and J.M. Cimbala, New York: McGraw-Hill, (2013).

10. A. N. Oumer, A., N. Hamidi, and I.M. Sahat. IOP Conf. Series: Mat.Sci. and Eng.(2015)

11. A. N. Oumer, and O. Mamat, Int. Jour. of Comp. Sci. and Eng., 9(3): 247-256 (2014).

12. A. N. Oumer, and O. Mamat, Compos. Part B: Eng.43(3): 1087-1094 (2012).

13. N. T. Rao., A. N. Oumer, and U.K. Jamaludin, The Jour. of Supercri. Flu., 116: 132-147 (2016).

14. Syavash. CFD online Fluent airfoil help (2012);

Avail. from:http://www.cfd-online.com/Forums/fluent/107547-fluent-airfoil-help.htmll\#post384545.

15. E. Robert. P. C. K. Sheldahl, 118, (1981). 